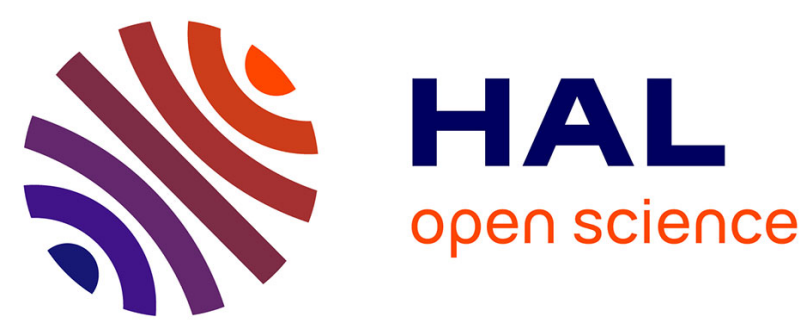

\title{
Electrically initiated upstream coalescence cascade of droplets in a microfluidic flow
}

M. Zagnoni, Charles N. Baroud, J.M. Cooper

\section{To cite this version:}

M. Zagnoni, Charles N. Baroud, J.M. Cooper. Electrically initiated upstream coalescence cascade of droplets in a microfluidic flow. Physical Review E: Statistical, Nonlinear, and Soft Matter Physics, 2009, 80 (4), pp.046303. 10.1103/physreve.80.046303 . hal-01021133

\section{HAL Id: hal-01021133 \\ https://hal-polytechnique.archives-ouvertes.fr/hal-01021133}

Submitted on 15 Jul 2014

HAL is a multi-disciplinary open access archive for the deposit and dissemination of scientific research documents, whether they are published or not. The documents may come from teaching and research institutions in France or abroad, or from public or private research centers.
L'archive ouverte pluridisciplinaire HAL, est destinée au dépôt et à la diffusion de documents scientifiques de niveau recherche, publiés ou non, émanant des établissements d'enseignement et de recherche français ou étrangers, des laboratoires publics ou privés. 


\title{
Electrically initiated upstream coalescence cascade of droplets in a microfluidic flow
}

\author{
Michele Zagnoni, ${ }^{1}$ Charles N. Baroud, ${ }^{2}$ and Jonathan M. Cooper ${ }^{1, *}$ \\ ${ }^{1}$ Department of Electronics \& Electrical Engineering, University of Glasgow, G12 8LT Glasgow, United Kingdom \\ ${ }^{2}$ Laboratoire d'Hydrodynamique (LadHyX) and Department of Mechanics, Ecole Polytechnique, F-91128 Palaiseau Cedex, France
}

(Received 18 May 2009; revised manuscript received 23 July 2009; published 2 October 2009)

\begin{abstract}
Two phase microfluidic systems creating size-controlled microdroplets have recently emerged as powerful tools to achieve liquid compartmentalization for high throughput chemical and biological assays. Emulsion electrocoalescence is a destabilization process that can be used in droplet-based platforms for water phase separation to enable lab-on-a-chip applications in biotechnology, including particle or cell recovery. In this paper, we report upon a series of phenomena associated with electrocoalescence of water microdroplet-in-oil populations in microfluidics. In our experiments, we formed microdroplets whose size and dispersion in the channel were varied according to the ratio of the flow rates of the two phases. Different types of electrocoalescence between droplets were obtained. For low applied voltages, drops merged in pairs over the electrode region; for higher values of the applied voltage, a cascade of droplet coalescence was produced against the flow direction, for a range of droplet sizes, lateral distributions of droplets in the channel and localized electric fields.
\end{abstract}

DOI: 10.1103/PhysRevE.80.046303

PACS number(s): 47.61.Jd, 47.55.df, 83.60.Np, 47.65.Gx

\section{INTRODUCTION}

Emulsion electrocoalescence is a process by which liquid droplets (i.e., the dispersed phase) immersed in an immiscible liquid medium (i.e., the continuous phase) merge as a consequence of an applied electric field [1-5]. This is an established technique in industrial applications employed to enhance the separation of water droplets in oil [6]: for example, either to remove aqueous dispersions from an organic phase for solvent extraction $[7,8]$ or in the petroleum industry to refine crude oil from water dispersions $[9,10]$.

Recently, microfluidic droplets have been proposed as a promising approach to lab-on-a-chip (LOC) applications, for instance, as mobile microreactors in which to perform the desired chemical or biological assays. In the LOC context, the merging of two drops would constitute one of the basic operations that need to be performed on microdroplets in order to achieve the potential capabilities. Indeed, it has already been reported that drops which may contain different reagents can flow alongside each other and be fused together at will through passive techniques [11,12], optical techniques [13], or electrical means. Electrocoalescence has emerged as a particularly useful tool to merge droplets in microfluidic channels, for example, by merging two populations of droplets with opposite charges [14] using electrodes aligned with a microchannel [15], combining the high potentials with hydrodynamic effects to bring together the droplets [16], or by merging microdroplets with a continuous water stream [17].

However, while many of the above reports demonstrate working devices which produce controlled electrocoalescence, the limits of the designs have remained less well defined, and it is not clear how the flow parameters affect the robustness of the operations. For instance, the effect of crowding in the microchannels remains unexplored although one would expect that the area fraction occupied by the dis-

*FAX: +44 131330 6010; jmcooper@elec.gla.ac.uk persed phase will play an important role. Moreover, there exist few systematic studies on the dependence of electrocoalescence on the actual value of the electric potential that is used.

In this paper, a systematic study of these two effects is presented using a defined oil-water system. The electrocoalescence of nanoliter and picoliter volume, surfactant stabilized water in oil drop populations in microfluidic channels is investigated, while varying the area fraction occupied by the water droplets and also the voltage that is used to induce the coalescence. These two parameters were found to play independent roles in addition to coupling together in producing a behavior in the form of cascades of droplet fusions upstream of the electrical forcing.

The electrocoalescence of drop populations and their upstream cascade are described as the experimental parameters are varied. We also provide evidence of coalescence mechanisms induced by a localized electric field for a stream of droplets in a microchannel.

\section{MATERIALS AND METHODS}

The continuous phase was composed of fluorocarbon oil (Fluorinert FC40, 3M) stabilized by surfactants: $0.5 \%$ wt Zonyl FSN-100 (Sigma-Aldrich) and 2\% wt Krytox 157FSL (Dupont). These solutions were sonicated for $20 \mathrm{~min}$ and used within $24 \mathrm{~h}$. The dispersed aqueous phase consisted of pure milli- $Q$ water.

Polydimethylsiloxane (PDMS) (Sylgard 184 Silicone Elastomer, Dow Corning) and glass substrates were used for the fabrication of the devices. Microchannels were fabricated in PDMS using standard soft lithography techniques. In order to create a silicon master, AZ4562 positive photoresist (Origine) was spin coated onto a silicon wafer at $4000 \mathrm{rpm}$ for $30 \mathrm{~s}$. After baking the substrate for $1 \mathrm{~min}$ at $90{ }^{\circ} \mathrm{C}$, the resist was exposed through a photomask to UV light for $16 \mathrm{~s}$ and was developed in a 1:4 ratio of AZ400K developer:water solution. The silicon wafer was subsequently dry etched, 


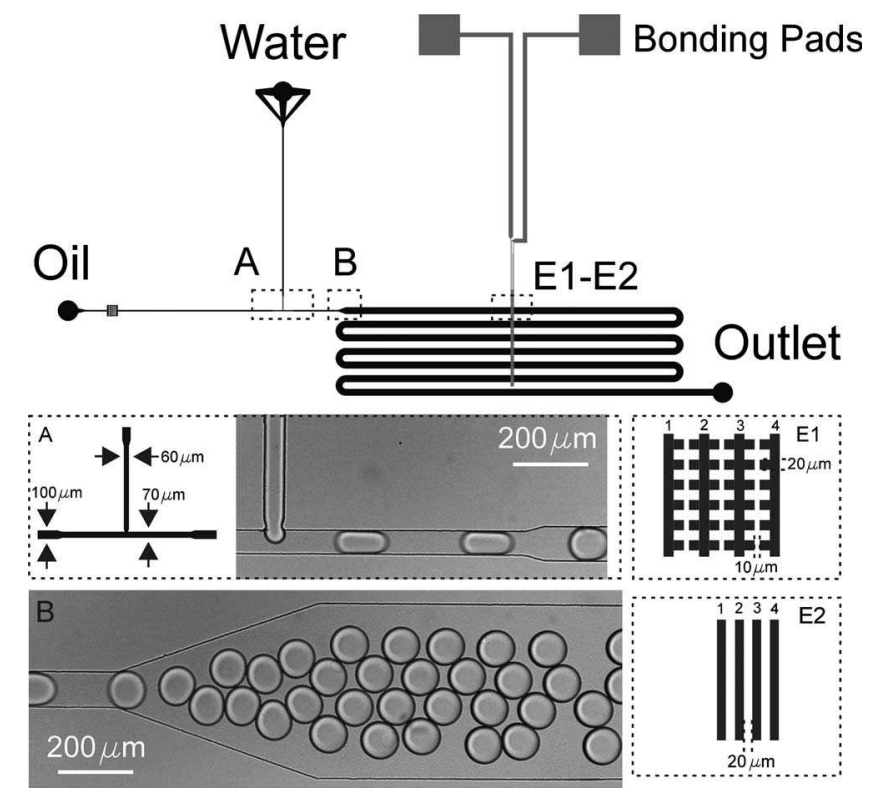

FIG. 1. Device geometry. A $T$ junction (dimensions as reported in inset A) was used to form water emulsions in oil. Further downstream, channel width is $500 \mu \mathrm{m}$ (inset B) and microelectrodes were placed perpendicular to the flow direction (dimensions and patterns as reported in inset $E_{1}$ and $E_{2}$ ). Bonding pads were used to contact wires from excitation source. A square waveform was applied at the electrodes, with $1-3$ and 2-4 sharing the same potentials.

achieving 50- $\mu \mathrm{m}$-deep patterns onto the master. To prevent PDMS adhesion to the master, the wafer was silanized using 1H-1H-2H-2H-perfluorooctyltrichlorosilane (Fluka) overnight. PDMS was then cast onto the silicon master at a 10:1 ratio of polymer to curing agent degassed in a desiccator chamber and cured at $70{ }^{\circ} \mathrm{C}$ overnight. The devices were then cut from the mold and punched with a sharpened flat needle to create inlet and outlet ports.

Microelectrodes were fabricated on a glass substrate using standard photolithography techniques, metal evaporation, and lift-off. The desired inverted electrode pattern was transferred into S1818 positive photoresist spun on a glass substrate (using an exposure time of $4.5 \mathrm{~s}$ ). A $10 \mathrm{~nm}$ titanium layer followed by a $60 \mathrm{~nm}$ gold layer were deposited onto the glass by electron-beam evaporation. The remaining photoresist was lifted off by washing in acetone.

Both PDMS and glass substrates were exposed to oxygen plasma, and the two parts of the chip were bonded together. Immediately after bonding, the device was flushed with undiluted Aquapel (PPG Industries, PA) and air to improve the wetting of the channels with fluorinated oil. PTFE (polytetrafluoroethylene) tubing with an inner diameter of $0.3 \mathrm{~mm}$ and an outer diameter of $0.76 \mathrm{~mm}$ (Cole Parmer) were used to connect the channels to syringes.

A schematic of the detailed device structure is shown in Fig. 1. A $T$ junction was used to create water emulsions in oil (inset A in Fig. 1). Further downstream, the droplets passed over a set of microelectrodes perpendicular to the channel (avoiding alignment steps and achieving reliable bonding). In each device, only one type of electrodes was used: either the configuration shown in the inset $E 1$ or in the inset $E 2$. There was no significant difference noted between the behavior during electrocoalescence between the two geometries (Fig. 1). Flow rates, ranging from $0.2-4 \mu \mathrm{L} / \mathrm{min}$ were generated and controlled by syringe pumps (New Era Pump System Inc.). Devices were placed over an inverted microscope and imaging was acquired using either a CoolSNAP HQ2 charge coupled device (CCD) camera (Photometrics) or a MotionScope M2 fast camera (Redlake). A TTi TG120 function generator was used to apply a signal to the electrodes using wires connected to the bonding pads. A pulsed dc field (with a square waveform of between $10 \mathrm{~Hz}$ to $1 \mathrm{MHz}$ and 0-20 V) was applied at the electrodes in the channel as shown in Fig. 1.

\section{EXPERIMENT}

Aqueous microdroplets in fluorocarbon oil were formed at a $T$ junction. Drop size was controlled by adjusting the flow rates of the continuous and dispersed phases [18], obtaining diameters varying from 65 to $300 \mu \mathrm{m}$. For the device geometry and the flow rates used in our work, the droplets never assumed a spherical geometry but were always constricted by the channel depth of $50 \mu \mathrm{m}$. Surfactants prevented droplets from coalescencing (both in static or dynamic flow condition) when no electric field was applied. Once the flow rates were stable (obtaining droplet size uniformity), different potential values $(0-20 \mathrm{~V})$ at different frequencies $(10$ $\mathrm{Hz}-10 \mathrm{MHz}$ ) were applied at the electrodes (inset $E_{1}$ and $E_{2}$ in Fig. 1). Droplet behavior was monitored at different ratios of the water (dispersed phase) and oil (continuous phase) flow rates while recording at different frame rate ranging from $15 \mathrm{~Hz}$ to $2 \mathrm{kHz}$. Image and data post processing was carried out using MATLAB.

\section{RESULTS AND DISCUSSION}

Before discussing the coalescence behavior of a droplet stream under a localized electric field, we describe the droplet flow and the effect of an electric field independently.

\section{A. Droplet stream behavior in the absence of electric field}

The behavior of a stream of droplets, flowing in the serpentine channel (500 $\mu \mathrm{m}$ width, $50 \mu \mathrm{m}$ depth), was characterized under different flow conditions without the application of an electric field. The total flow rate $Q_{T}$ is the sum of the continuous phase flow rate $Q_{C}$ and the dispersed phase flow rate $Q_{D}$.

Figure 2 shows mean droplet area and area fraction $F$ (representative of the lateral distribution of the droplets in the channel and calculated as the area of the dispersed phase over the microchannel area in the field of view). Droplet stream images are shown in Figs. 2(a)-2(c) as $Q_{D}, Q_{C}$, and $Q_{T}$ were varied, while the average droplet area and the area fraction $F$ are shown in Figs. 2(d) and 2(e), for values of $Q_{D} / Q_{T}$ from 0.25 to $0.75 . F$ is expected to be equal to $Q_{D} / Q_{T}$ in the case of perfectly two-dimensional drops, since the flow rates of the oil and water phases are fixed and con- 

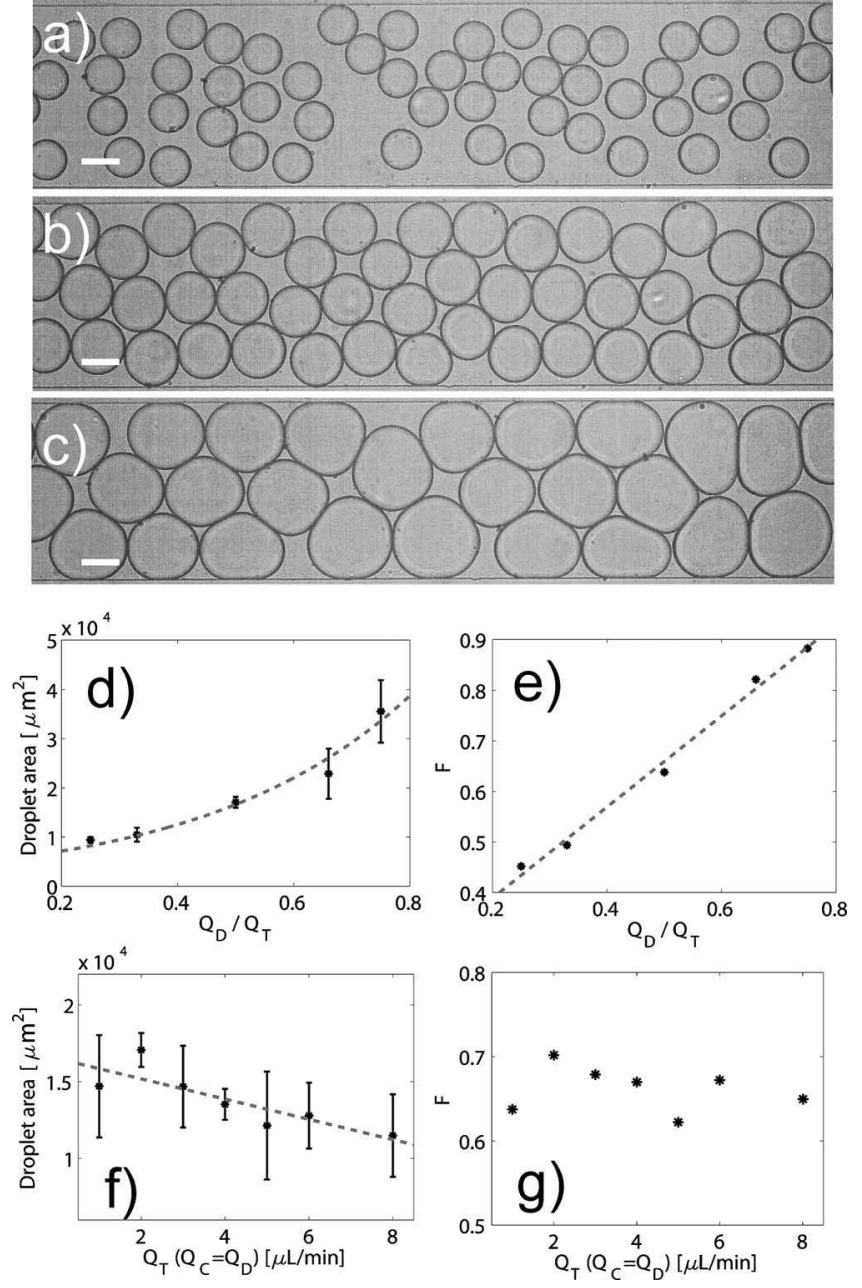

FIG. 2. Emulsions flowing from left to right inside the serpentine channel: (a) $Q_{C}=3 \mu \mathrm{L} / \mathrm{min}, Q_{D}=1 \mu \mathrm{L} / \mathrm{min}$; (b) $Q_{C}$ $=1 \mu \mathrm{L} / \mathrm{min}, Q_{D}=1 \mu \mathrm{L} / \mathrm{min} ; \quad$ (c) $Q_{C}=0.5 \mu \mathrm{L} / \mathrm{min}, Q_{D}$ $=1.5 \mu \mathrm{L} / \mathrm{min}$. [(d) and (e)] Average droplet area and area fraction, $F$, for different values of $Q_{D} / Q_{T}: 0.25\left(Q_{D}=1 \mu \mathrm{L} / \mathrm{min}, Q_{C}\right.$ $=3 \mu \mathrm{L} / \mathrm{min}), 0.33\left(Q_{D}=1 \mu \mathrm{L} / \mathrm{min}, Q_{C}=2 \mu \mathrm{L} / \mathrm{min}\right), 0.5\left(Q_{D}\right.$ $\left.=1 \mu \mathrm{L} / \mathrm{min}, \quad Q_{C}=1 \mu \mathrm{L} / \mathrm{min}\right), \quad 0.66 \quad\left(Q_{D}=2 \mu \mathrm{L} / \mathrm{min}, \quad Q_{C}\right.$ $=1 \mu \mathrm{L} / \mathrm{min})$, and $0.75\left(Q_{D}=1.5 \mu \mathrm{L} / \mathrm{min}, Q_{C}=0.5 \mu \mathrm{L} / \mathrm{min}\right)$. Dashed lines are fitting the data with an exponential function (d) and a line (e) using a least square algorithm. [(f) and (g)] Average droplet area and area fraction $F$ of water to oil for $Q_{C}=Q_{D}$ and $Q_{T}=1,2,3,4,5,6$, and $8 \mu \mathrm{L} / \mathrm{min}$, respectively (dashed line is fitting the data with a line using a least square algorithm). Scale bar is $100 \mu \mathrm{m}$.

servation of volume must be maintained for each of the fluids. However, we find that $F$ is affine with respect to $Q_{D} / Q_{T}$, displaying a slope of 0.92 , which can be explained by considering several different possible sources of experimental uncertainty (including small variations in channel geometry and flow rate as well as the curvature of the drops and the effect of the oil lubrication films between the microchannel walls and the drops). Nonetheless, $F$ displays a near linear dependence on $Q_{D} / Q_{T}$ which is useful in interpreting the results of the electrofusion experiments. For a fixed $Q_{D}$, the larger $Q_{T}$, the smaller the droplet area and the smaller the area fraction, $F$. As the emulsions increase in size, they oc- cupy the majority of the channel, as also clearly evident from the Figs. 2(a)-2(c).

When $Q_{C}$ and $Q_{D}$ were held equal and the total flow rate $Q_{T}$ was varied from 1 to $8 \mu \mathrm{L} / \mathrm{min}$, little variation in droplet area and area fraction $F$ was observed, as shown in Figs. 2(f) and $2(\mathrm{~g})$ (with a minor decrease in both parameters for increasing values of $Q_{T}$ ). This showed that difference in droplet size was almost negligible when $Q_{C}=Q_{D}$, in agreement with theory and experimental results reported in [18].

Moreover, the thickness of the carrier fluid layer between droplets became progressively thinner as the drop size increased. This is observed by comparing the mean interdroplet distance $D_{\mathrm{ID}}$ versus the mean drop diameter $D_{\mathrm{MD}}$. Both are extracted from droplet images, with $D_{\text {ID }}$ calculated as the mean distance between the center of mass of a drop and the center of mass of each droplet surrounding it, while $D_{\mathrm{MD}}$ is computed as the square root of the drop area normalized by $\pi$. These parameters are shown in Figs. 3(a)-3(c) for different flow conditions. The ratio between these two parameters $\left(D_{\mathrm{ID}} / D_{\mathrm{MD}}\right)$ is also shown with respect to the droplet area (proportional to $Q_{D} / Q_{T}$, as shown in Fig. 2).

For increasing $Q_{D} / Q_{T}$, both $D_{\mathrm{ID}}$ and $D_{\mathrm{MD}}$ increased, though at different rates [Fig. 3(c)]: the more the droplet size decreased, the more the oil layer that surrounded the droplets increased in thickness. This implies that droplets flowing at larger values of $Q_{D} / Q_{T}$ are more likely to be touching other drops and to be deformed from their circular state, as shown by the values of $D_{\mathrm{ID}} / D_{\mathrm{MD}} \leq 1$.

Finally, the average droplet velocity inside the channel is shown in Fig. 3(d) for $Q_{C}=Q_{D}$ and $Q_{T}$ from 1 to $8 \mu \mathrm{L} / \mathrm{min}$. Results show that the average droplet velocity can be approximated as the total nominal flow rate divided by the cross section of the channel.

\section{B. Electric field description in a two phase system}

Electric fields interact with droplets by inducing forces on the water-oil interface. The field lines will also be deviated by the physical contrast between the drop and the carrier fluid. This coupled interaction depends on the difference in conductivity and permittivity between the two fluids [1-5] in addition to the details of the geometry and the distance between drops in the case of dense two-phase flows. The effects can be described by the Taylor-Melcher leaky dielectric model which states that free charges can accumulate on the interface between the droplet and the outer fluid, thus inducing a tangential and a normal Maxwell stresses at this boundary between the drop and surrounding fluid [19,20].

This stress leads to viscous "Stokes" flow in and around the drop, which in turn yields both a net body force and a deformation of the drop to accommodate the electrical and viscous stresses [21]. The electrical force can cause macroscopic effects over the droplet according to the electric field strength by deforming, slowing down, and even trapping a flowing droplet over the electrodes [20,22].

Further interactions occur due to the presence of surfactant molecules in solution which, in addition to reducing the surface tension between droplets and the carrier fluid, also stabilize emulsions and prevent drop coalescence $[23,24]$. An 
a)

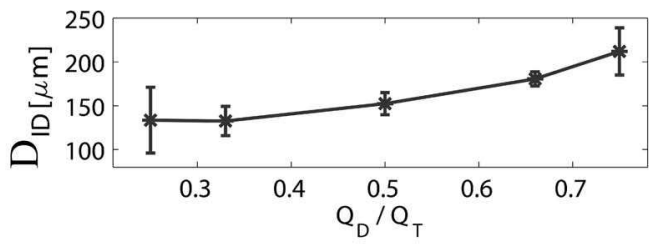

b)

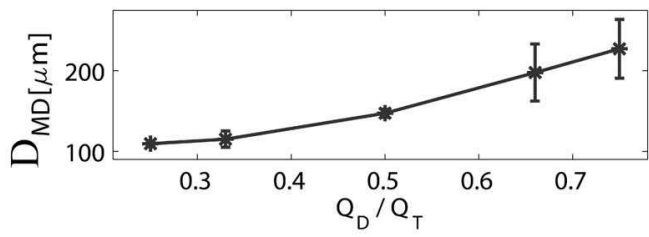

c)

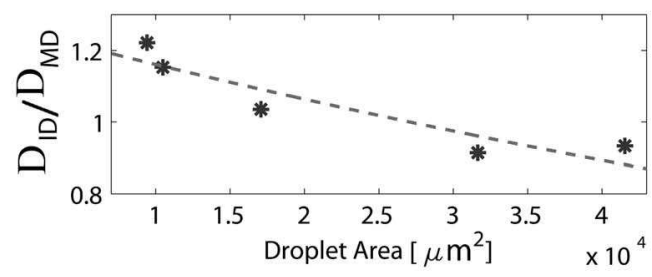

d)

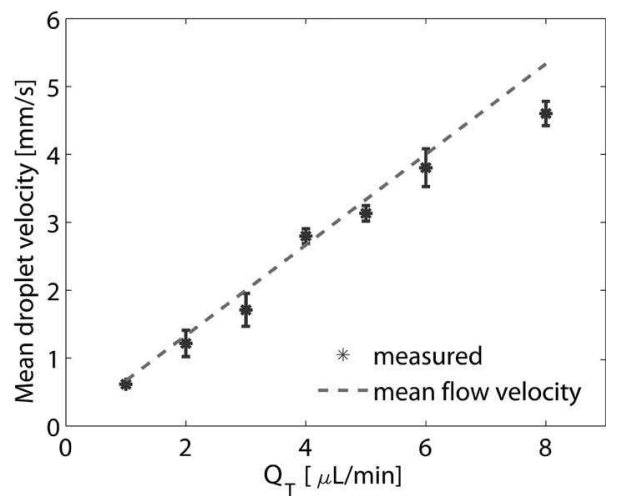

FIG. 3. (a) Mean interdroplet distance $\left(D_{\text {ID }}\right)$ and (b) mean droplet diameter $\left(D_{\mathrm{MD}}\right)$ for different values of $Q_{D} / Q_{T}: 0.25\left(Q_{D}\right.$ $\left.=1 \mu \mathrm{L} / \mathrm{min}, \quad Q_{C}=3 \mu \mathrm{L} / \mathrm{min}\right), \quad 0.33 \quad\left(Q_{D}=1 \mu \mathrm{L} / \mathrm{min}, \quad Q_{C}\right.$ $=2 \mu \mathrm{L} / \mathrm{min}), 0.5\left(Q_{D}=1 \mu \mathrm{L} / \mathrm{min}, Q_{C}=1 \mu \mathrm{L} / \mathrm{min}\right), 0.66\left(Q_{D}\right.$ $\left.=2 \mu \mathrm{L} / \mathrm{min}, Q_{C}=1 \mu \mathrm{L} / \mathrm{min}\right)$, and $0.75\left(Q_{D}=1.5 \mu \mathrm{L} / \mathrm{min}, Q_{C}\right.$ $=0.5 \mu \mathrm{L} / \mathrm{min}$ ). (c) ratio $D_{\mathrm{ID}} / D_{\mathrm{MD}}$ over the droplet area (dashed line is fitting the data with a line using a least square algorithm). (d) Average droplet velocity inside the serpentine channel for $Q_{C}=Q_{D}$ and $Q_{T}=1,2,3,4,5,6$, and $8 \mu \mathrm{L} / \mathrm{min}$, respectively. Mean flow velocity was calculated by dividing $Q_{T}$ for the cross-sectional area of the channel.

effect of the electric field is to displace or realign the polar surfactant molecules along the field lines, thus competing with the chemical potential which would tend to align them normal to the interface $[25,26]$. This rearrangement of surfactant can lead to variations in surface tension but also can deplete certain zones of the drop surface. When two drops are in close proximity and subjected to an electric field, the complex interactions of the field deformation, the surfactant dynamics, and the interface shape can lead to the destabilization of the drop interfaces which can be bridged together, leading to the rapid fusion of the two drops. This "electrofusion" has been the subject of a wide literature in several fields [14-16,23,27].

In our design, the electric field was generated by the micropatterned electrodes as described above. Contrary to other

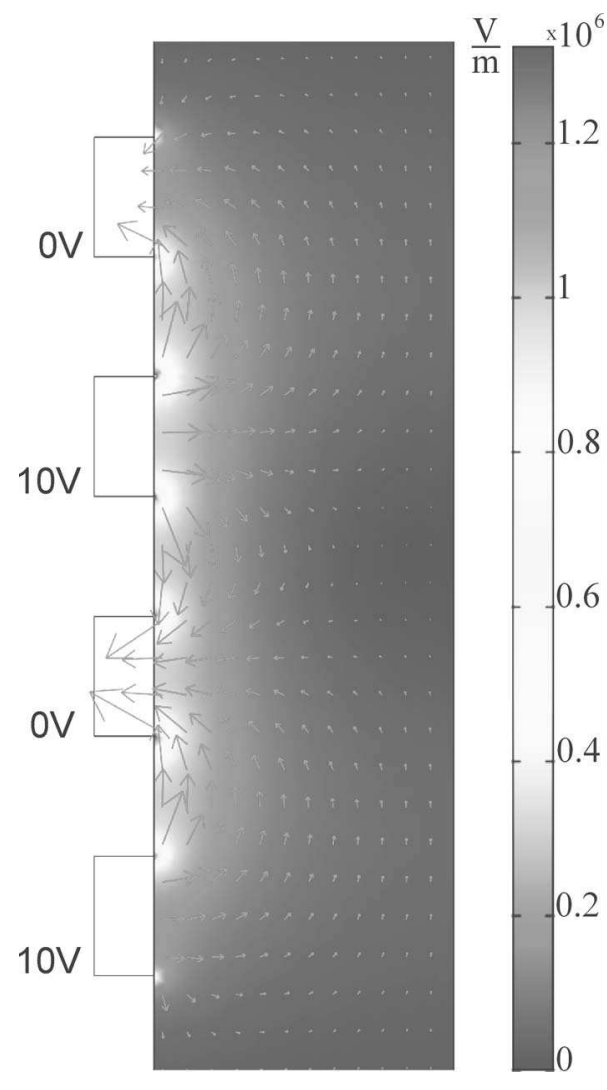

FIG. 4. Numerical simulations obtained using COMSOL 3.3. Side view of the electric field distribution inside the channel around the electrode area for a pulsed dc square wave applied at the electrodes (potential values reported in the figure).

systems, the applied electric field is localized to regions that are smaller or comparable to the drop dimension (the electrode spacing being 10-20 $\mu \mathrm{m})$. The field is nonuniform even in the absence of the drops as shown by a finite-element calculation in Fig. 4. Large field values appear near the edges of the electrode pattern and field lines connect the positive and negative electrodes through the bulk fluid within the channel. The magnitude of the field varies between 0 and 13 $\mathrm{kV} / \mathrm{cm}$ on a spatial scale of $20 \mu \mathrm{m}$ (the distance between the electrodes) and the field vanishes to $1 \%$ of the maximum value a distance variable from $20 \mu \mathrm{m}$ from the end electrodes. The presence of a water droplet will strongly modify the field lines, focusing them close to the oil-water interface and shielding other regions, in a manner that varies with the drop position and shape $[1,19]$.

\section{DROPLET STREAM BEHAVIOR IN THE PRESENCE OF ELECTRIC FIELD}

For the flow rates and liquids used in our study, four different behaviors of electrocoalescence were observed when a stream of droplets passed over the electrodes, as shown in Fig. 5 (movie1 in [28]). These depended not only upon the magnitude and the frequency of the applied square wave but also upon the droplet distribution in the channel. The behaviors were found to be independent of the geometry of the electrodes used in this paper. 


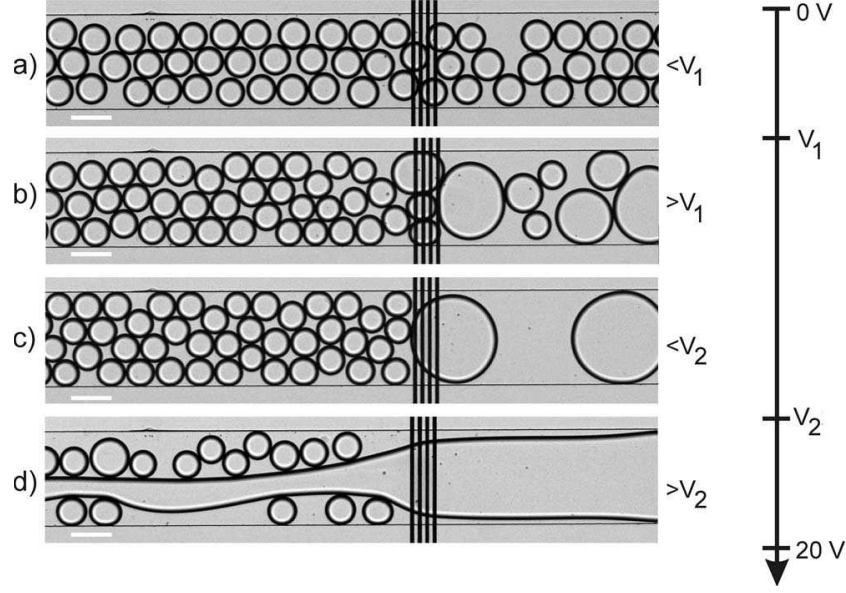

FIG. 5. Electrocoalescence behaviors of a stream of water droplets in oil under the effect of an applied pulsed dc field at a frequency of $10 \mathrm{kHz}$ : (a) no coalescence occurred without the application of subthreshold electric fields; (b) droplet coalescence originated when a potential threshold $V_{1}$ was reached; (c) increasing the potential led to drop coalescence that spanned the channel width; (d) drop coalescence is propagated against flow direction when a potential threshold $V_{2}$ was reached. Scale bar is $200 \mu \mathrm{m}$.

Qualitatively, when the applied potentials were below a threshold value $V_{1}$, the electric field effect was not strong enough to induce coalescence and the droplets did not merge while flowing past the electrodes [Fig. 5(a)]. For potential values equal or just above the threshold $V_{1}$, electrocoalescence occurred only for droplets that, while flowing, were positioned in a region between the electrodes.

As shown in Fig. 5(b), not every drop merged with another: this depended on the magnitude of the electric field and on the distance, $D_{\mathrm{ID}}$. When the potential was further increased, every droplet passing over the electrode area merged, resulting in larger drops that spanned the entire width of the channel [Fig. 5(c)], confirming that the presence of coalescence strongly depended upon the local magnitude of the electric field and on the relative positions of the drops. Finally, when the applied potentials were above a higher threshold $V_{2}$, the initial fusion event near the electrodes propagated upstream of the electrode region [Fig. 5(d)] through a cascade of droplet merging events that resulted in a "finger" growing against the flow direction and then retreating at irregular intervals (movie2 in [28]).

These four different behaviors were observed under different conditions of droplet area and lateral distribution in the channel, which were varied by adjusting the continuous and dispersed phase flow rates. As previously shown in Figs. 2 and 3 , for a fixed total flow rate $Q_{T}$, the higher the flow rate of the carrier fluid $\left(Q_{C}\right)$ with respect to the water $\left(Q_{D}\right)$, the smaller the droplet size formed at the $T$ junction and the larger the separation between droplets, thus influencing the droplet distribution in the channel. Experimentally, droplet coalescence upstream of the electrode region only occurred in the range $0.25 \leq Q_{D} / D_{T} \leq 0.75$. When $Q_{D} / Q_{T}<0.25$, droplets were on average separated from others by thick layers of carrier fluid as shown in Fig. 2. When $Q_{D} / Q_{T}>0.75$, on the other hand, single droplets spanned almost half the
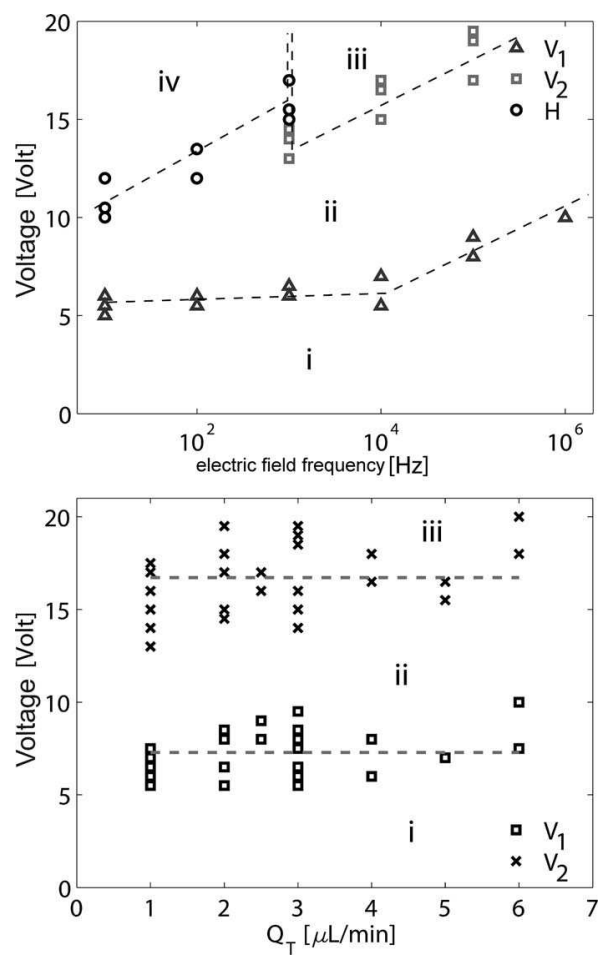

FIG. 6. (Top) Droplet electrocoalescence behavior as a function of the electric field frequency for total flow rates. $Q_{T}=3 \mu \mathrm{L} / \mathrm{min}$ and $Q_{D} / Q_{T}=0.33 \mu \mathrm{L} / \min \left(Q_{D}=1, Q_{C}=2\right), 0.5 \mu \mathrm{L} / \min \left(Q_{D}\right.$ $\left.=1.5, Q_{C}=1.5\right)$, and $0.66 \mu \mathrm{L} / \mathrm{min}\left(Q_{D}=2, Q_{C}=1\right)$. Four regions were identified: (i) no coalescence, (ii) downstream coalescence, (iii) upstream coalescence, and (iv) hydrolysis. (Bottom) Droplet electrocoalescence results from over 30 experiments where $Q_{T}$ varied from 1 to $6 \mu \mathrm{L} / \mathrm{min}$, the ratio $Q_{D} / Q_{T}$ varied from 0.28 up to 0.7 and the frequency was fixed at $10 \mathrm{kHz}$. Dashed lines indicate mean values. The following values were calculated for a distance between electrodes of $20 \mu \mathrm{m}$ (inset $E_{2}$ in Fig. 1): obtaining a mean value of $7.24 \mathrm{~V}$ for $V_{1}$ and $16.1 \mathrm{~V}$ for $V_{2}$, which convert in electric field strength values of 3.62 and $8.05 \mathrm{kV} / \mathrm{cm}$, respectively. At 10 $\mathrm{kHz}$ hydrolysis never occurred and region of type (i)-(iii) were obtained.

width of the channel and drops were tightly packed together, limiting fluid motion in the zone of influence of the electrodes.

Figure 6 shows quantitative information concerning coalescence behaviors. In order to investigate the dependence of droplet coalescence upon the electric field frequency, we explored the circumstance in which $Q_{T}$ was fixed at $3 \mu \mathrm{L} / \mathrm{min}$ and the amplitude of a pulsed dc square wave was ramped between 0 and $20 \mathrm{~V}$ for frequencies between $10 \mathrm{~Hz}$ and 1 $\mathrm{MHz}$. This was repeated for different ratio of continuous and dispersed phase: $Q_{D} / Q_{T}=0.33, Q_{D} / Q_{T}=0.5$, and $Q_{D} / Q_{T}$ $=0.66$. The results are summarized in Fig. 6(a). Four regions were identified: (i) no coalescence occurred when the potential was below a threshold $V_{1}$ for every frequency tested; (ii) coalescence of droplet was observed for every frequency tested in a region comprised between a threshold $V_{1}$ and a higher threshold given either by $\mathrm{H}$ for lower frequencies (where hydrolysis was obtained) or by $V_{2}$ for higher frequencies (where droplet coalescence upstream the electrodes was obtained); (iii) droplet coalescence occurred against the flow 
direction at irregular intervals; (iv) hydrolysis was observed as well as droplet coalescence and the experiment was stopped. As indicated by dashed lines in Fig. 6(a), threshold behavior $\left(V_{1}, V_{2}\right.$, ad $\left.\mathrm{H}\right)$ depended upon the applied frequency, following a general trend: the lower the frequency, the lower the threshold value.

Considering water permittivity $\varepsilon_{\mathrm{W}}=80$ and conductivity $\sigma_{\mathrm{W}}=2-10 \mu \mathrm{S} / \mathrm{m}$ (measured), the dipole relaxation-time constant $\tau=\varepsilon_{0} \varepsilon_{\mathrm{W}} / \sigma_{\mathrm{W}}$ varies from 300 to less than $70 \mu \mathrm{s}$. These values confirm that, for the frequencies tested and for the droplet velocities reported in Fig. 3, the electric field always produced an effect over the droplets that traveled over the electrode region between 20 and $140 \mathrm{~ms}$. No relevant difference was observed for different ratios of $Q_{D} / Q_{T}$ if the frequency was varied.

Figure 6(b) shows the results in which $Q_{T}$ was between 1 and $6 \mu \mathrm{L} / \mathrm{min}$ and $Q_{D} / Q_{T}$ varied between 0.28 and 0.7. No defined trend was observed that suggested a relation between the coalescence behavior and $Q_{T}$ or $Q_{D} / Q_{T}$ (the transition behavior of $V_{2}$ in the range $Q_{D} / Q_{T}=0.25-0.28$ and $0.7-0.75$ has not been studied). However, results showed that the electric field magnitude had to be substantially more than twice the value that initiated coalescence in order to observe droplet merging upstream from the electrodes. The results also showed that a critical field strength is necessary to induce droplet coalescence against the flow direction when certain conditions of droplet size and distribution in a microchannel were satisfied.

\section{UPSTREAM COALESCENCE}

The upstream coalescence mechanism was investigated by analyzing the process at a frame rate of $2 \mathrm{kHz}$ (movie3 in [28]), as shown in Fig. 7. The process of upstream coalescence proceeded in two steps: the first consisted of two or more water drops that suddenly merged near the electrode area [Figs. 7(f) and 7(g)]. This initial fusion always happened when the interface between the two drops was upstream of the electrodes, which was only possible when the electric field magnitude was above $V_{2}$. We refer to this type of fusion as "electric effect" (EE) fusion. In the second step, this initial fusion induced a cascade of droplet coalescence events that propagated upstream at a rate much higher than the flow velocity in the microchannel [Figs. $7(\mathrm{~h})-7(\mathrm{t})]$. The physics of this second stage is dominated by the hydrodynamic rearrangement of the liquid interfaces through capillary action and we will refer to it as "hydrodynamic effect" (HE) fusion. Hereafter, these two aspects are analyzed, separately.

\section{A. Electric effect fusion}

Figures $8(a)$ and $8($ b) show two different examples in which upstream droplet coalescence was initiated by a strong electric field $\left(V>V_{2}\right)$. In both cases, coalescence took place between two drops whose interface had not yet reached the electrodes. At this interface, the thin oil film separating the two droplets was initially stabilized by the presence of a surfactant film at the interface [29]. We propose that the elec-

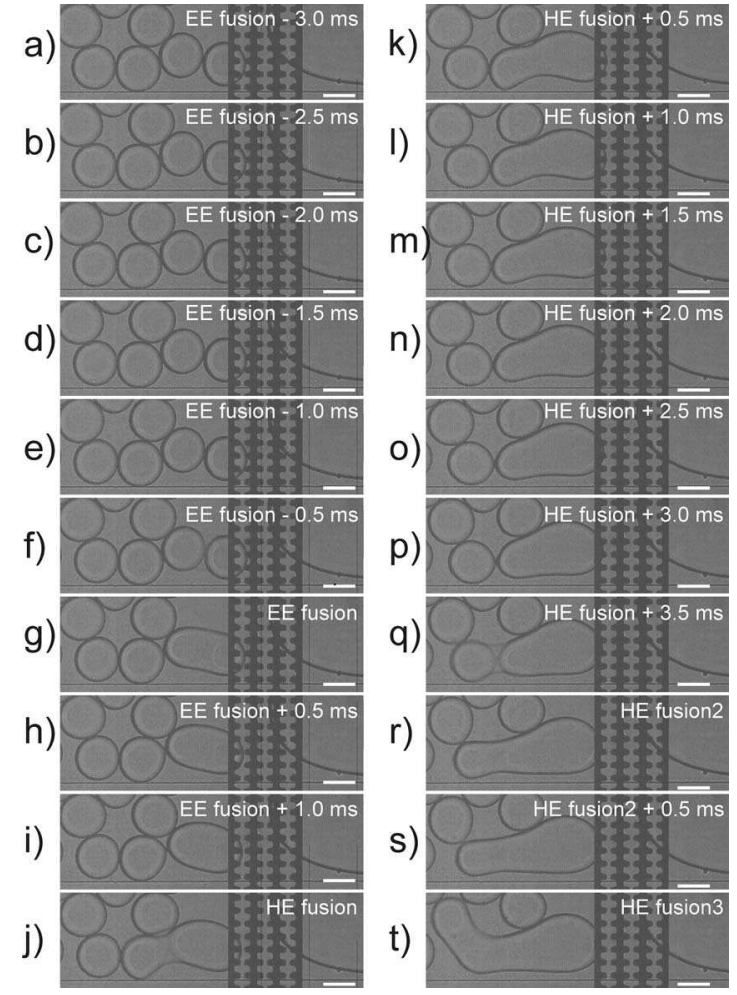

FIG. 7. Example of a time sequence showing upstream coalescence induced in a microchannel (sequence was recorded at $2 \mathrm{kHz}$ ). Two different types of fusion were observed in upstream coalescence: $[(\mathrm{a})-(\mathrm{g})]$ EE fusion caused only by the electric field strength; [(h)-(t)] HE fusion caused only by localized decompressions and capillary forces between a droplet and the retreating finger. Scale bar is $100 \mu \mathrm{m}$.

tric field is capable, in these cases, of reorienting the surfactant molecules by exerting an electric force over their dipoles as is the case for cell electroporation [30] or when rupturing a lipid bilayer [26,27].

When the applied voltage is higher than the second threshold $V_{2}$, the electric field propagates sufficiently far from the electrode area and is able to cause electric breakdown of the oil-surfactant interface between two droplets: the oil-surfactant film ruptures due to the electric field magnitude (for $V>V_{2}$ ) before the two interfaces reach the electrodes.

This has significant consequences on the shape of the merged droplet: immediately after an EE fusion, the new drop is no longer circular, with some regions having significantly larger curvature from the main body of the droplet, as shown in Fig. 8. This implies a strong imbalance in the pres-

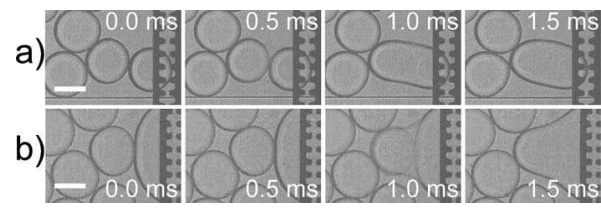

FIG. 8. [(a) and (b)] Two time sequences of droplet fusion induced by the EE fusions. No droplet deformation was observed before fusion. 
a)
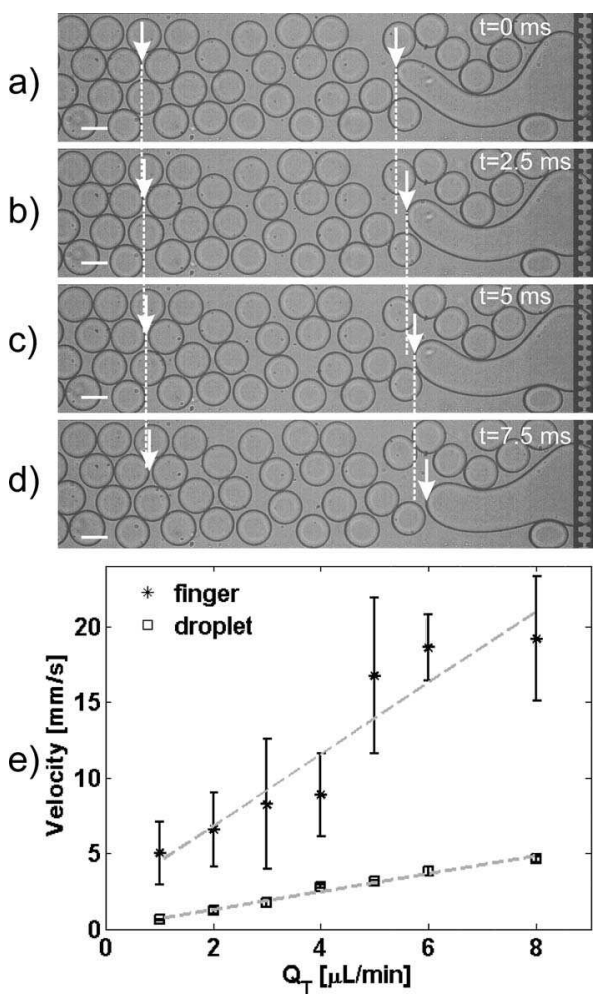

FIG. 9. [(a)-(d)] Time sequence showing an aqueous finger moving toward the electrodes at a velocity higher than the droplet stream. It can be noticed (white dashed lines and arrows) that the finger shortened along the flow direction and, at the same time, became wider along the width of the channel. This effect was caused by surface tension, the finger wanting to minimize the surface in contact with the oil. (e) Average velocity of aqueous finger and droplet for total flow rate, $Q_{T}=1,2,3,4,5,6$, and $8 \mu \mathrm{L} / \mathrm{min}$. Scale bar is $100 \mu \mathrm{m}$.

sure field inside the drop due to the variations in the Laplace pressure jump, which in turn leads to a rapid retraction of the newly formed interface from the regions of high curvature toward regions of low curvature.

\section{B. Hydrodynamic effect fusion}

The shape rearrangement of two drops (initiated by EE fusion) with respect to the adjacent drops was found to produce a cascade of merging events through successive fluid decompressions. The retraction of the interface of a newly formed finger creates a differential velocity between the finger and the surrounding droplets: a droplet found in the proximity of the finger is then attracted toward the finger interface and a new fusion can occur (depending on the parameters $D_{\mathrm{ID}}$ and $D_{\mathrm{MD}}$ in Fig. 3).

A comparison between the motions of a finger with that of a drop far upstream is shown in Figs. 9(a)-9(d). In this particular experiment, none of the adjacent droplets were close enough $\left(D_{\mathrm{ID}}\right)$ to be influenced by the finger and no coalescence occurred. Figure 9(e) shows a comparison between the average velocity of the finger tip and of a droplet in a stream for different values of $Q_{T}$. For each flow rate, the finger front a)
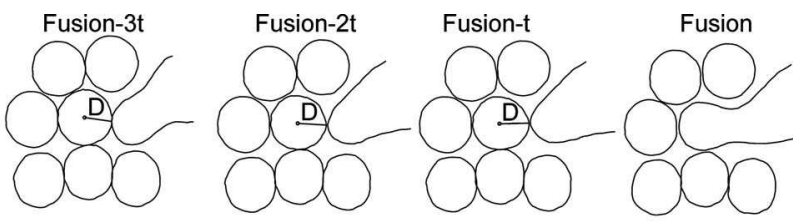

b)

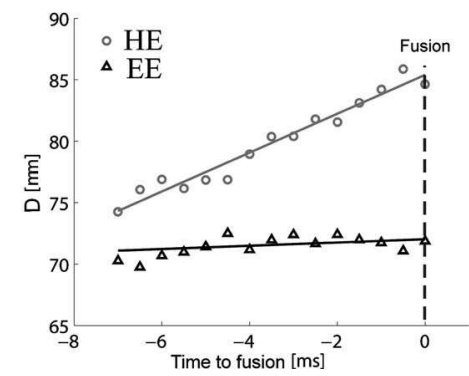

FIG. 10. (a) Schematic of a time sequence of droplet fusion where $Q_{D}=1$ and $Q_{C}=2$. The parameter $D$ is the semi axis of a droplet obtained as the distance between the center of mass of a droplet and the interface of the droplet along the fusion direction and it represents droplet deformation. (b) Examples of evolution of the distance $D$ versus time for a droplet fusing in the first stage (triangles) and second stage (circles) of upstream coalescence. The rightmost of each sequence corresponds to the last frame before fusion occurred. $D(t)$ has a gradient of $0.03 \mu \mathrm{m} / \mathrm{ms}( \pm 0.1 \mathrm{std}, n$ $=10)$ in the moments before fusion in the first case (EE). When coalescence occurred in the second case (HE), $D$ increased with time, yielding a gradient of $1.29 \mu \mathrm{m} / \mathrm{ms}( \pm 0.34 \mathrm{std}, n=10)$.

moved toward the electrodes at least five times faster than a droplet advanced.

This counterintuitive mechanism, by which two drops merge as they are moving apart, has already been observed under several different circumstances [11,12,31]. It has been interpreted as resulting from a significant reduction in pressure in the lubricating film that separates the two interfaces when the distance between them increases. Indeed, the continuous phase must replenish the gap between the two drops as they separate but the resistance to flow in the confined space requires a decrease in the local pressure. In some cases, this pressure drop was found to strongly deform the water interfaces and to create pointed structures on the water-oil surface [11]. However, the details of the mechanism remain only partially clear, with surfactant depletion at the drop interface also playing a role [31].

Droplet deformation was measured in our experiments prior to $\mathrm{EE}$ and $\mathrm{HE}$ fusion events, and two representative examples are reported in Fig. 10 for similarly sized droplets. The deformation is represented by the distance $D$ defined as the distance between the center of mass of a droplet and the interface of the finger (or drop) in the direction of fusion, as shown in Fig. 10(a). The distance $D$ between drops, in the case of EE fusion, does not show any clear trend prior to merging, while droplets appear to deform linearly before merging within the HE regime. Indeed, the value of $D(t)$ has a negligible gradient in the moments before fusion in the first case (EE). When coalescence occurred in the second case (HE), however, $D$ increased significantly with time until fusion occurred. Repetition of this initial phenomenon subsequently produced an upstream cascade as the fusion- 
retraction process was propagated from one droplet to another.

These results confirm once more that the fusion mechanisms in the two cases are different. While hydrodynamic forces played no significant role in drop fusion in the first case, they were the only forces responsible to produce the cascade. In addition, upstream coalescence was always initiated with an EE fusion, which produced a fluid condition capable of triggering a decompression wave.

\section{SUMMARY AND DISCUSSION}

In this paper, electrocoalescence of monodisperse droplet populations in a microchannel has been investigated. Droplet coalescence was studied as the electrical and fluid parameters were varied. The effect of changing the flow rates was to modify the lateral distribution of drops and their size, with a clear dependence of droplet area and packing as the oil and water flow rates are varied. The electrical field frequency did not have a noticeable effect on the behavior of the merging of drops. However, two threshold voltages were found: the first determines the minimum value for fusion to occur between two adjacent droplets; the second leads to a cascade of merging events that rapidly propagate upstream of the electrode region.

The upstream cascade can be described as a two-stage mechanism: the first stage, which is dominated by electric effects, occurs in regions near the electrodes where fusion is triggered solely by the electric field (voltage about $15 \mathrm{~V}$ and above). For these high values of the electric field, the initial fusion event took place sufficiently upstream of the electrodes to produce a strong imbalance in the curvature along the drop interface. This imbalance produced a rapid retraction of the newly formed finger and led to the second stage of the cascade in which a series of localized decompressions occurred among the finger and the next drop causing them to merge together. This produces an aqueous finger that propa- gated against the flow direction through successive fusion events as it retracted downstream through the action of surface tension. In order to obtain upstream coalescence, it was necessary for the first fusion (as in Fig. 8) to occur in a region of the channel where the magnitude of the electric field was capable of breaking down the oil-surfactant interface, but not able to deform the drop interface or to limit fluid motion considerably (as in the area directly above the electrodes where drops undergo deformation [32]).

As shown above, upstream coalescence depended on droplet size and distribution, these two parameters influencing the rearrangement of the carrier fluid around the droplets. This accounted for the fact that no upstream coalescence manifested neither when $Q_{D} / Q_{T}>0.75$, where droplet size limited fluid motion nor when $Q_{D} / Q_{T}<0.25$, where the layer of oil surfactant between droplets was too thick and could not be broken down by the electric field. Finally, upstream coalescence only occurred in dynamic conditions. When an electric potential was applied to static emulsions inside the channel, coalescence occurred only over the electrode area (movie4 in [28]).

Although previous work has generally concentrated on producing localized and well controlled coalescence between drops, cascades of drop mergings can potentially offer different uses in LOC technologies. For instance, they can be used to rapidly extract large sample volumes, for example, in order to "reset" the parameters of an experiment. This behavior can also lead to major variations in the flow properties when many small drops are rapidly replaced with a long finger which has smaller hydrodynamic resistance. Such rearrangements can have an important effect on the pressure balance in a connected microfluidic network of channels.

\section{ACKNOWLEDGMENT}

This work was supported by BBSRC (Grant No. BB/ F005024/1).
[1] M. Chabert, K. D. Dorfman, and J. L. Viovy, Electrophoresis 26, 3706 (2005).

[2] J. S. Eow, M. Ghadiri, and A. Sharif, Colloids Surf., A 225, 193 (2003)

[3] J. S. Eow and M. Ghadiri, Colloids Surf. A 219, 253 (2003).

[4] P. Atten, J. Electrost. 30, 259 (1993).

[5] J. S. Eow and M. Ghadiri, Chem. Eng. J. 85, 357 (2002).

[6] J. S. Eow, M. Ghadiri, A. O. Sharif, and T. J. Williams, Chem. Eng. J. 84, 173 (2001).

[7] P. J. Bailes and S. K. L. Larkai, Trans. IChemE, Part C 59, 229 (1981).

[8] P. J. Bailes and S. K. L. Larkai, Trans. IChemE, Part C 60, 115 (1982).

[9] F. G. Cottrell and J. B. Speed, U. S. Patent No. 987,115 (21 March, 1911).

[10] F. G. Cottrell, U. S. Patent No. 987,114 (21 March, 1911).

[11] N. Bremond, A. R. Thiam, and J. Bibette, Phys. Rev. Lett. 100, 024501 (2008).
[12] X. Niu, S. Gulati, J. B. Edel, and A. J. deMello, Lab Chip 8, 1837 (2008).

[13] C. N. Baroud, M. R. de Saint Vincent, and J. P. Delville, Lab Chip 7, 1029 (2007).

[14] D. R. Link, E. Grasland-Mongrain, A. Duri, F. Sarrazin, Z. Cheng, G. Cristobal, M. Marquez, and D. A. Weitz, Angew. Chem., Int. Ed. 45, 2556 (2006).

[15] C. Priest, S. Herminghaus, and R. Seemann, Appl Phys Lett. 89, 134101 (2006).

[16] K. Ahn, J. J. Agresti, H. Chong, M. Marquez, and D. A. Weitz, Appl. Phys. Lett. 88, 264105 (2006).

[17] L. M. Fidalgo, G. Whyte, D. Bratton, C. F. Kaminski, C. Abell, and W. T. S. Huck, Angew. Chem., Int. Ed. 47, 2042 (2008).

[18] P. Garstecki, M. J. Fuesterman, H. A. Stone, and G. M. Whitesides, Lab Chip 6, 437 (2006).

[19] P. Singh and N. Aubry, Electrophoresis 28, 644 (2007).

[20] P. M. Young and K. Mohseni, ASME J. Fluids Eng. 130, 081603 (2008). 
[21] D. A. Saville, Annu. Rev. Fluid Mech. 29, 27 (1997).

[22] J. C. Baygents, N. J. Rivette, and H. A. Stone, J. Fluid Mech. 368, 359 (1998).

[23] T. F. Tadros, Applied Surfactants: Principles and Applications (Wiley-VCH, New York, 2005)

[24] G. F. Christopher and S. L. Anna, J. Phys. D 40, R319 (2007).

[25] P. J. Bowen and T. J. Lewis, Thin Solid Films 99, 157 (1983).

[26] T. J. Lewis, IEEE Trans. Dielectr. Electr. Insul. 10, 769 (2003).

[27] S. Aghdaei, M. E. Sandison, M. Zagnoni, N. G. Green, and H.
Morgan, Lab Chip 8, 1617 (2008).

[28] See EPAPS Document No. E-PLEEE8-80-160909 for movie1, movie2, movie3, and movie4. For more information on EPAPS, see http://www.aip.org/pubservs/epaps.html.

[29] J. Bibette, F. Leal Calderon, and P. Poulin, Rep. Prog. Phys. 62, 969 (1999).

[30] E. Neumann, S. Kakorin, and K. Toensing, Bioelectrochem. Bioenerg. 48, 3 (1999).

[31] B. Dai and L. G. Leal, Phys. Fluids 20, 040802 (2008).

[32] M. Zagnoni and J. M. Cooper, Lab Chip 9, 2652 (2009). 